T. Ohsawa

Nagoya Math. J.

Vol. 147 (1997), 107-112

\title{
ON THE STABILITY OF PSEUDOCONVEXITY FOR CERTAIN COVERING SPACES
}

\author{
TAKEO OHSAWA
}

\begin{abstract}
It is proved that, if $X \stackrel{\pi}{\rightarrow} T$ is a proper holomorphic map with one-dimensional fibers and $\tilde{X} \stackrel{\varpi}{\longrightarrow} X$ a covering map, a point $t \in T$ has a neighbourhood $U$ such that $\varpi^{-1}\left(\pi^{-1}(U)\right)$ is holomorphically convex if and only if $\varpi^{-1}\left(\pi^{-1}(t)\right)$ is holomorphically convex.
\end{abstract}

$\S 1$. It has long been known that the parameter space of a complex analytic family of complex manifolds often admits a significant geometric structure that deserves intensive studies. To describe such a structure, theory is extremely useful when the fibers are Riemann surfaces, because any family is then transformed into that of quasifuchsian groups in $\operatorname{PSL}(2, \mathbf{C})$. In higher dimensions, however, the uniformization theory has not been developed enough to capture important aspects of the variation of complex structures. Nevertheless it seems natural to expect that covering spaces somehow carry information about the deformation. Thus we would like to continue the previous work [O-2] on the holomorphic convexity of the covering spaces of a family of compact Riemann surfaces over the unit disc.

\$2. First we ask for the possibility of constructing a plurisubharmonic function on the covering of the family by extending one from the special fiber. We shall give an answer to this question which is almost trivial (see Theorem 1). However, as we shall see after Theorem 2, this poses an interesting question on the extension of bounded plurisubharmonic functions. Next we would like to extend the result in [O-2] to the case of families with singular fibers. It will turn out that the absence of infinite chain of compact complex curves is sufficient for the holomorphic convexity at the germ level (cf. Theorem 3).

Received November 27, 1995. 
$\S 3$. Let $T$ be any contractible complex space, let $X$ be a complex space with a proper and surjective holomorphic map $\pi: X \rightarrow T$, and let $t_{0}$ be any point of $T$. We put $X_{t}=\pi^{-1}(t)$ for $t \in T$ and $X_{U}=\pi^{-1}(U)$ for $U \subset T$. Let $\varpi: \tilde{X} \rightarrow X$ be any covering space. For simplicity we put $\tilde{X}_{t}=\varpi^{-1}\left(X_{t}\right)$ and $\tilde{X}_{U}=\varpi^{-1}\left(X_{U}\right)$.

$\S 4$. First we study the case where $X$ and $T$ are nonsingular and $\pi$ is everywhere of maximal rank. It is remarkable that $\tilde{X}_{t}$ are not necessarily Stein even if $\tilde{X}_{t_{0}}$ is biholomorphically equivalent to $\mathbf{C}^{n}$ (cf. [N]). In contrast to this phenomenon we are going to give a criterion for the stability of Steinness. Under the above situation, we fix a neighbourhood $U_{1} \ni t_{0}$ and a $C^{\infty}$ retraction $r: X_{U_{1}} \rightarrow X_{t_{0}}$. Furthermore let $\tilde{r}: \tilde{X}_{U_{1}} \rightarrow \tilde{X}_{t_{0}}$ be the lift of $r$.

THEOREM 1. Let $\psi$ be a $C^{\infty}$ plurisubharmonic function on $\tilde{X}_{t_{0}}$. Suppose that there exists a Hermitian metric $g$ on $X_{t_{0}}$ such that the complex Hessian of $\psi$ is larger than $\varpi^{*} g$ and that the covariant derivatives of $\psi$ with respect to $\varpi^{*} g$ are bounded up to the second order. Then, for any strictly plurisubharmonic function $\varphi$ on $T$ there exists a neighbourhood $U \ni t_{0}$ such that $\psi \circ \tilde{r}+\varphi \circ \pi \circ \varpi$ is strictly plurisubharmonic on $\tilde{X}_{U}$.

Proof. In terms of a local expression $\left(r_{1}, \ldots, r_{n}\right)$ of $\tilde{r}$ with respect to a holomorphic local coordinate $\left(z_{1}, \ldots, z_{n}\right)$ of $\tilde{X}_{t_{0}}$,

$$
\begin{aligned}
\partial \bar{\partial}(\psi \circ r)= & \partial \bar{\partial} \psi\left(r_{1}, \ldots, r_{n}\right)=\partial\left(\sum_{k} \frac{\partial \psi}{\partial z_{k}} \bar{\partial} r_{k} \sum_{k} \frac{\partial \psi}{\partial \bar{z}_{k}} \bar{\partial} \bar{r}_{k}\right) \\
= & \sum_{j, k} \frac{\partial^{2} \psi}{\partial z_{j} \partial z_{k}} \partial r_{j} \bar{\partial} r_{k}+\sum_{j, k} \frac{\partial^{2} \psi}{\partial z_{j} \partial \bar{z}_{k}} \partial \bar{r}_{j} \bar{\partial} r_{k}+\sum_{k} \frac{\partial \psi}{\partial z_{k}} \partial \bar{\partial} r_{k} \\
& +\sum_{j, k} \frac{\partial^{2} \psi}{\partial \bar{z}_{j} \partial z_{k}} \partial \bar{r}_{j} \bar{\partial} r_{k}+\sum_{j, k} \frac{\partial^{2} \psi}{\partial \bar{z}_{j} \partial \bar{z}_{k}} \partial \bar{r}_{j} \bar{\partial} \bar{r}_{k}+\sum_{k} \frac{\partial \psi}{\partial z_{k}} \partial \bar{\partial} \bar{r}_{k} .
\end{aligned}
$$

The desired conclusion follows from the boundedness of the derivatives of $\psi$ and that $\left(\partial^{2} \psi / \partial z_{j} \partial \bar{z}_{k}\right)>0, \bar{\partial} r_{k}=\partial \bar{r}_{j}=\partial \bar{\partial} r_{k}=\partial \bar{\partial} \bar{r}_{k}=0$ on $\tilde{X}_{t_{0}}$.

This shows that the pseudoconvexity property of covering spaces is stable under some hyperbolicity condition, which is certainly not satisfied by $\mathbf{C}^{n}$. 
COROLlARY. Under the hypothesis of Theorem 1, suppose moreover that $\psi$ is an exhaustion function, i.e. all the sublevel sets of $\psi$ are relatively compact in $\tilde{X}_{t_{0}}$. Then there exists a neighbourhood $U \ni t_{0}$ and a $C^{\infty}$ plurisubhamonic functions $\varphi$ on $U$ such that $\psi \circ r+\varphi \circ \pi \circ \varpi$ is a strictly plurisubharmonic exhaustion function on $\tilde{X}_{U}$. In particular, $\tilde{X}_{U}$ is a Stein manifold for such a neighbourhood $U \ni t_{0}$.

$\S 5$. The following is also an immediate consequence of Theorem 1 , but we want to state it separately as a theorem because of an interesting question it poses.

Theorem 2. Let $\pi: X \rightarrow T$ be everywhere of maximal rank. If there is a biholomorphic map

$$
\sigma:\{z \in \mathbf{C} ;|z|<1\} \rightarrow \tilde{X}_{t_{0}}
$$

then for any $\eta \in(0,1)$ there exists a neighbourhood $U \ni t_{0}$ and a negative plurisubharmonic function $\Phi_{\eta}$ on $X_{U}$ such that $\Phi_{\eta}(z)=-\left(1-|z|^{2}\right)^{\eta}$ and $\pi \circ \varpi \mid\left\{x ; \Phi_{\eta}(x) \leq c\right\}$ is proper for all $c<0$.

Proof. After extending the function $\left(\sigma^{-1}\right)^{*}\left(-\log \left(1-|z|^{2}\right)\right.$ to a saturated neighbourhood $\tilde{X}_{U}$ by Theorem 1 , compose $-\exp (-\eta t)$ to it.

We note that $-\log \left(1-|z|^{2}\right)$ satisfies the condition of Theorem 1 because it is a potential of the Bergman metric whose covariant derivatives (up to order two) are all bounded.

$\S$. From now on we allow $X_{t_{0}}$ to have singular points. The main achievement of the present note is the following.

THEOREM 3. If $\operatorname{dim} X_{t_{0}}=1$ and $\tilde{X}_{t_{0}}$ is holomorphically convex, then there exists a neighbourhood $U \ni t_{0}$ such that $\tilde{X}_{U}$ is holomorphically convex.

Proof. We may assume that $\tilde{X}_{t_{0}}$ is noncompact since the conclusion is trivial otherwise. Moreover we may assume that $X_{t_{0}}$ is connected since it suffices to show the holomorphic convexity for each connected component of $\tilde{X}_{U}$ for some neighbourhood $U \ni t_{0}$. Since $\tilde{X}_{t_{0}}$ is holomorphically convex, the union of compact irreducible components of $\tilde{X}_{t_{0}}$ consists of compact 
connected components, say $\left\{C_{\alpha}\right\}_{\alpha \in A}$. Since any of these components intersects with noncompact irreducible components, there exists a complex space $\hat{X}$ and a proper holomorphic map $f: \tilde{X} \rightarrow \hat{X}$ such that for each $\alpha \in A f\left(C_{\alpha}\right)$ consists of a single point and $f \mid \tilde{X}_{t_{0}} \backslash \cup_{\alpha \in A} C_{\alpha}$ is a biholomorphism. Therefore it suffices to show that $f\left(\tilde{X}_{U}\right)$ is a Stein space for some neighbourhood $U \ni t_{0}$. We shall prove it by extending an increasing family of open subsets $\left\{R_{j}\right\}_{j \in \mathbf{N}}$ of $f\left(\tilde{X}_{t_{0}}\right)$ to its neighbourhood of the form $f\left(\tilde{X}_{U}\right)$ so that the extension $\left\{R_{j}^{*}\right\}_{j \in \mathbf{N}}$ satisfies

$$
\left(R_{j}^{*}, R_{j+1}^{*}\right) \text { is a Runge pair for every } j
$$

and

$$
f\left(\tilde{X}_{U}\right)=\bigcup_{j=1}^{\infty} R_{j}^{*} .
$$

For that, let us take a $C^{\infty}$ strictly subharmonic exhaustion function on $f\left(\tilde{X}_{t_{0}}\right)$. Such a function exists since $f\left(\tilde{X}_{t_{0}}\right)$ contains no compact irreducible components. In fact, it is proved in $[\mathrm{O}-1]$ in a more general setting that given any discrete subset containing all the singular points of $f\left(\tilde{X}_{t_{0}}\right)$, say $\Gamma$, and any strictly subharmonic function $p$ defined on a neighbourhood $W \supset \Gamma$ such that $p \mid \Gamma$ is exhaustive, one can find a $C^{\infty}$ function $\tilde{p}: f\left(\tilde{X}_{t_{0}}\right) \rightarrow \mathbf{R}$, a neighbourhood $W^{\prime} \supset \Gamma$ with $W^{\prime} \subset W$, and a $C^{\infty}$ convex increasing function $\lambda: \mathbf{R} \rightarrow \mathbf{R}$ such that $p=\tilde{p}$ on $W^{\prime}$ and $\lambda \circ \tilde{p}$ is a strictly subharmonic exhaustion function. On the other hand, since $\operatorname{dim} X_{t_{0}}=1$, there exist a finite set $\left\{x_{1}, \ldots, x_{m}\right\} \subset X_{t_{0}}$ and arbitrarily small disjoint neighbourhoods $V_{j} \ni x_{\jmath}$ in $X$ such that one can find a Stein neighbourhood $U \ni t_{0}$ and a holomorphic retraction from $X_{U} \backslash \cup_{j=1}^{m} V_{j}$ onto $X_{t=0} \backslash \cup_{j=1}^{m} V_{j}$, say $\kappa$. The lift of $\kappa$ to $\tilde{X}_{U} \backslash \varpi^{-1}\left(\cup_{j=1}^{m} V_{j}\right)$ will be denoted by $\tilde{\kappa}$. Let $Y$ be the union of noncompact components of $\varpi^{-1}\left(X_{t_{0}} \backslash \cup_{j=1}^{m} V_{j}\right)$ and let $Y_{U}=\tilde{\kappa}^{-1}(Y)$. Note that $f \mid Y_{U}$ is biholomorphic, so that we may identify $Y_{U}$ with $f\left(Y_{U}\right)$. Shrinking $V_{j}$ if necessary, one has an exhaustive strictly subharmonic function $q$ on $f\left(\tilde{X}_{t_{0}}\right)$ such that the ranges of $q$ on the connected components $Z_{k}, k \in \mathbf{N}$, of $f\left(\tilde{X}_{t_{0}}\right) \backslash Y$, are mutually disjoint, and that $q \mid \partial Z_{k}=$ const and $d q \mid \partial Z_{k}$ has no zeroes for every $k$. Let $c_{k}=\sup _{Z_{k}} q$ and $d_{k}=\inf _{Z_{k}} q$. Then we put

$$
\begin{aligned}
& R_{k}^{\prime}=\left\{x ; q(x)<d_{k}\right\} \\
& R_{k}^{\prime \prime}=R_{k}^{\prime} \cup Z_{k}
\end{aligned}
$$




$$
\begin{aligned}
R_{k}^{\prime \prime \prime} & =\left\{x ; q(x)<c_{k}\right\} \\
R_{3 j-2} & =R_{j}^{\prime} \\
R_{d j-1} & =R_{j}^{\prime \prime} \\
R_{3 j} & =R_{j}^{\prime \prime \prime}
\end{aligned}
$$

and let $R *_{j}$ be an open subset of $f\left(\tilde{X}_{U}\right)$ satisfying $R_{j}^{*} \cap f\left(\tilde{X}_{t_{0}}\right)=R_{j}$ and $\partial R_{j}^{*}=\tilde{\kappa}^{-1}\left(\partial R_{j}\right)$. By a criterion of Grauert and Narasimhan (cf. [G], [Nr]), it is readily seen that $R_{j}^{*}$ are all Stein spaces. Furthermore $\left(R_{3 j-1}^{*}, R_{3 j}^{*}\right)$ are Runge pairs, since the open sets $R_{s}^{*}$ defined by $R_{s}^{*} \cap f\left(\tilde{X}_{t_{0}}\right)=\{x ; q(x)<s\}$ and $\partial R_{s}^{*}=\tilde{\kappa}^{-1}(\{x ; q(x)=s\})$, for $c_{k}<s<d_{k}$, provides a semicontinuous holmorphic extension (halbstetige holomorphe Ausdehnung) $\left\{R_{s}^{*} \cup R_{3 j-1}^{*}\right\}$ from $R_{3 j-1}^{*}$ to $R_{3 j}^{*}$ in the sense of Docquier-Grauert [D-G].

We recall that an open subset $D$ of a complex manifold $X$ admits a semicontinuous holomorphic extension to $X$ if there exists an increasing family of open subsets $\left\{D_{t}\right\}_{0 \leq t \leq 1}$ of $X$ such that

1) $D_{t}$ is Stein for almost all $t$.

2) $D_{0}=D, \bigcup_{t<1} D_{t}=X$.

3) $\bigcup_{0 \leq t<t_{0}} D_{t}=D_{t_{0}}$ for all $t_{0}$.

4) $\left(\bigcap_{t_{0}<t} D_{t}\right)^{o}=D_{t_{0}}$ for all $t_{0}$.

Here $A^{o}$ denotes the set of interior points of $A$.

Similarly $\left(R_{3 j}^{*}, R_{3 j+1}^{*}\right)$ are Runge pairs for all $j$. That $\left(R_{3 j-2}^{*}, R_{3 j-1}^{*}\right)$ is a Runge pair is trivial. Thus $f\left(\tilde{X}_{U}\right)$ is a Stein space, so that $\tilde{X}_{U}$ is holomorphically convex.

Acknowledgement The author is grateful to Prof. Masahide Kato who evoked his attention to Nakamura's example in [N]. He also thanks the referee for criticisms.

\section{REFERENCES}

[D-G] F. Docquier and H. Grauert, Levisches Problem und Rungescher Satz für Teilgebiete Steinscher Mannigfaltıgkeiten, Math. Ann., 140 (1960), 94-123. 
[G] H. Garuert, On Levi's problem and the imbedding of real-analytic manifolds, Ann. of Math., 68 (1958), 460-472.

[N] I. Nakamura, On complex parallelisable manifolds and their small deformations, Proc. Japan Acad., 48 (1972), 447-449.

[Nr] R. Narasimhan, The Levi problem for complex spaces II, Math. Ann., 146 (1962), 195-216.

[O-1] T. Ohsawa, Completeness of noncompact analytic spaces, Publ. RIMS, Kyoto Univ., 20 (1984), 683-992.

[O-2] T. Ohsawa, A note on the variation of Riemann surfaces, Nagoya Math. J., 142 (1996), 1-4.

Graduate School of Polymathematics

Nagoya University

Chikusa-ku, Nagoya $464-01$

Japan

ohsawa@math.nagoya-u.ac.jp 